\title{
Stars, Star Clusters, and Dust in NGC 3077
}

\author{
T. J. Davidge ${ }^{12}$ \\ Canadian Gemini Office, Herzberg Institute of Astrophysics, \\ National Research Council of Canada, 5071 West Saanich Road, \\ Victoria, B.C. Canada V9E 2E7 \\ email: tim.davidge@nrc-cnrc.gc.ca
}

\begin{abstract}
Images obtained with the CFHTIR camera on the Canada-France-Hawaii Telescope are used to investigate the near-infrared photometric properties of the star-forming M81 group galaxy NGC 3077. The spectral-energy distribution (SED) of the near-infrared light within 10 arcsec of the nucleus (1) is very different from that of 'typical' dwarf ellipticals (dEs), blue compact dwarf galaxies, and HII/starburst galaxies, and (2) is consistent with the $2 \mu \mathrm{m}$ light being dominated by hot young $\left(\log \left(\mathrm{t}_{y r}\right)<6.8\right)$ stars reddened by $\mathrm{A}_{V}=3-4$, with $\mathrm{A}_{V} \geq 8 \mathrm{mag}$ in some regions, including previously detected areas of $\mathrm{CO}$ emission. A population like that near the center of NGC 205 likely contributes only a modest fraction of the light near $2 \mu \mathrm{m}$.

A number of candidate star clusters are detected in and around NGC 3077. These objects have near-infrared brightnesses and colors that are consistent with them being classical globular clusters and young star clusters. The specific frequency of globular clusters in NGC 3077 is estimated to be $\mathrm{S}_{N}=2.5$, which falls within the range of $\mathrm{S}_{N}$ measured in nearby dEs. The candidate young clusters have photometric masses that are similar to those of compact young clusters in other active star-forming systems, and SEDs consistent with ages $\log \left(\mathrm{t}_{y r}\right) \leq 6.6$. Based on the masses and ages of the young clusters, it is estimated that the star formation rate in NGC 3077 was at least $0.25-0.50 \mathrm{M}_{\odot}$ year $^{-1}$ during the past few million years.
\end{abstract}

Subject headings: galaxies: individual (NGC 3077) - galaxies: evolution galaxies: dwarf

\footnotetext{
${ }^{1}$ Visiting Astronomer, Canada-France-Hawaii Telescope, which is operated by the National Research Council of Canada, the Centre National de la Recherche Scientifique, and the University of Hawaii

${ }^{2}$ This publication makes use of data products from the Two Micron All Sky Survey, which is a joint project of the University of Massachusetts and the Infrared Processing and Analysis Center/California Institute of Technology, funded by NASA and the NSF.
} 


\section{INTRODUCTION}

The origin of dwarf spheroidal (dSph) and dwarf elliptical (dE) galaxies, and their relation to dwarf irregular (dIrr) galaxies, has long been a matter of debate (e.g. Tajiri \& Kamaya 2002; Mayer et al. 2001; Silk, Wyse, \& Shields 1987; Thuan 1985; Lin \& Faber 1983). At issue is whether the morphological characteristics of these galaxies are primarily the result of (1) local conditions within the mini-halos from which they first collapsed, or (2) external factors, such as proximity to a much larger companion. Numerical simulations predict that the initial density and dark matter content are critical parameters for defining final morphology (e.g. Dekel \& Silk 1986; Ferrara \& Tolstoy 2000; Carraro et al. 2001), and the correlation between the chemical compositions, central surface brightness, and mass-to-light ratios of a wide range of dwarf galaxies is consistent with initial conditions playing a key role in dwarf galaxy evolution (Prada \& Burkert 2002). However, the occurence of morphological segregation in nearby groups (e.g. Karachentsev et al. 2002) and the relation between gas content and distance from nearby large companions in the Local Group (Blitz \& Robishaw 2000) indicate that environment also plays a key role in dwarf galaxy evolution. It is also clear that tidal interactions can profoundly affect the structural properties of dwarf galaxies in hierarchical systems, with the Sagitarrius dwarf being a prime example (Ibata, Gilmore, \& Irwin 1995).

Studies of nearby galaxies and their companions will provide insights into dwarf galaxy evolution. The Milky-Way, M31, and M81 have similar masses (e.g. Kochanek 1996; Schroder et al. 2002; Perrett et al. 2002), morphologies, and environments, and yet have very different satellite systems. The companions of the Milky-Way include the dIrr Magellanic Clouds and a number of dSphs, while the brightest companions of M31 are the dE galaxies NGCs 147, 185, and 205, and the compact elliptical galaxy M32; M31 also has a number of fainter dSph satellites. The companions of M81 show considerable diversity, consisting of dSphs, dEs, and dIrrs, and some of these show morphological peculiarities that are related to tidal interactions. Indeed, the M81 group appears to be undergoing significant evolution at the present day, making this the nearest laboratory for studying the effects of on-going tidal interactions on gas-rich dwarf galaxies.

NGC 3077 is an actively star-forming member of the M81 group that is not easily placed within the Hubble sequence, although Price \& Gullixson (1989) conclude that there are similarities with the Local Group dE NGC 185 at red wavelengths. There is a prominent central dust lane, and filamentary $\mathrm{H} \alpha$ emission (Barbieri, Bertola, \& di Tullio 1974). The optical depth of the central dust lane is $\sim 0.5$, and this absorption obscures the isophotal center of the galaxy at visible wavelengths (Price \& Gullixson 1989). The central regions of NGC 3077 are dominated by hot stars (Benacchio \& Galletta 1981), and the star formation 
rate in the central 700 parsecs exceeds that in normal discs (e.g. Thronson, Wilton, \& Ksir 1991; Ott, Martin, \& Walter 2003). Martin (1998) identified a number of expanding gas shells in the ISM of NGC 3077. These structures have kinematic ages $\leq 10 \mathrm{Myr}$, and spatial scales and expansion velocities that are indicative of energy input from a large number of SNe, thus confirming that there has been considerable recent star-forming activity.

NGC 3077 is not evolving in isolation. Ott et al. (2003) conclude that while the hot gas in NGC 3077 is confined at present, some of it may eventually escape into the M81 group intergalactic medium. An HI bridge connects M81 and NGC 3077 (van der Hulst 1979), while tidal spurs also link NGC 3077 to other members of the M81 group (e.g. Boyce et al. 2001). The interactions with M81 and its companions likely spurred the current star-forming episode in NGC 3077, and may also have triggered the formation of young compact star clusters in the M81 disk (Chandar et al. 2001).

Studies of stars and star clusters in NGC 3077 will provide insight into the past history of this galaxy. The central regions of NGC 3077 have yet to be resolved into stars. However, Sakai \& Madore (2001) and Karachentsev et al. (2002) resolved stars on the upper RGB in the outer regions of NGC 3077, and the latter conclude that $\mu_{0}=27.91$, placing the system behind M81. The presence of RGB stars indicates that NGC 3077 is not a recently formed tidal fragment, as has been suggested for some other M81 companions (e.g. Karachentsev, Karachentseva, \& Boerngen 1985; Yun, Ho, \& Lo 1994; Boyce et al. 2001).

In the present study, deep $J, H$, and $K^{\prime}$ images obtained with the CFHTIR camera are used to probe the near-infrared spectral energy distribution (SED) and morphology of the central regions of NGC 3077, and search for star clusters. Observations of this galaxy in the infrared are of interest because the evolved cool stars that formed during intermediate and early epochs might be expected to dominate the light output at these wavelengths; knowledge of the spatial distribution of such stars may yield insight into the nature of NGC 3077 prior to the most recent tidal interactions, and provide clues about its appearance after the current star-forming episode fades. Light at infrared wavelengths is also less affected by dust absorption than at visible wavelengths, making it easier to probe the heavily obscured central regions of NGC 3077, and detect bright star clusters.

The paper is structured as follows. The observations and the data reduction procedures are described in $\S 2$. The infrared SED of integrated light in the central regions of NGC 3077 and the isophotal properties of the galaxy are investigated in $\S 3$. Comparisons are also made with the Local Group dE galaxy NGC 205, which shows some similarities with NGC 3077, and may be in a more advanced evolutionary state (Davidge 1992). While the present data do not have the angular resolution needed to detect individual stars, a number of potential star clusters are identified, and the nature of these is investigated in 
$\S 4$. A summary and discussion of the results follows in $\S 5$.

\section{OBSERVATIONS AND REDUCTIONS}

The data were recorded on June 42001 UT with the CFHTIR imager, which was mounted at the Cassegrain focus of the 3.6 metre Canada France Hawaii Telescope (CFHT). The detector in the CFHTIR is a $1024 \times 1024 \mathrm{Hg}$ :Cd:Te array. Each pixel subtends 0.2 arcsec on a side, so that a $3.4 \times 3.4$ arcmin field is imaged.

Images of NGC 3077 were recorded through $J, H$, and $K^{\prime}$ filters with a total exposure time of $360 \mathrm{sec}$ per filter. A four point square dither pattern was employed to assist with the identification of cosmic-rays and bad pixels, and to facilitate the construction of on-sky calibration frames. Faint standard stars from Hawarden et al. (2001) were also observed. The standard deviation of the $K$ zeropoints measured from individual stars is \pm 0.04 magnitudes. A comparison with sources in the 2MASS Point Source Catalogue confirms that the photometric calibration is reliable to within a few hundredths of a magnitude $(\S 4.1)$.

Each image was processed using the following sequence: (1) the subtraction of a master dark frame, which was constructed by combining dark frames taken with the same integration time as the science observation, (2) the division by a dome flat, which was constructed by taking the difference between images of a dome spot recorded with the dome lights on and off to remove thermal artifacts produced by warm objects along the line of sight, (3) the subtraction of the DC sky level, and (4) the subtraction of thermal artifacts and interference fringes from the on-sky images. The calibration images used in the last step were constructed from flat-fielded and sky-subtracted images of different fields, which were median-combined to reject stars and galaxies. The processed images were aligned to correct for the offsets in the dither pattern, and then median-combined to reject cosmic rays and bad pixels. The processed $K^{\prime}$ image of NGC 3077, trimmed to the area of common integration time, and with the diffuse body of the galaxy, a template for which was obtained by median filtering the final processed image, subtracted out to allow sources near the galaxy center to be seen, is shown in Figure 1. Individual sources in the final combined images have a FWHM of 0.7 arcsec, and so the spatial resolution of these data, based on the Sakai \& Madore (1999) distance estimate, is roughly 13 parsecs. 


\section{THE CENTRAL REGIONS OF NGC 3077}

\subsection{The Appearance and SED in the Near-Infrared}

The central $12 \times 14 \operatorname{arcsec}$ of NGC 3077 in $K^{\prime}$ is shown in the right hand panel of Figure 2, while a gray scale map of the $J-K$ color in this same region is shown in the left hand panel of this figure. The sky levels used to construct the color map were measured near the edges of the CFHTIR field in an effort to reduce contamination from the outer regions of the galaxy, and the maximum extent of the region shown in Figure 2 was selected to have a surface brightness that was high enough to be immune to the estimated uncertainties in the measured background level. Evidence that the photometric properties in this portion of NGC 3077 are not greatly affected by uncertainties in the sky level comes from the well-behaved nature of the $K$ surface brightness profile out to 20 arcsec radius, and the absence of systematic color gradients in this same region (§3.3).

Images at visible wavelengths show dust lanes across the face of NGC 3077 (Barbieri et al. 1974; Price \& Gullixson 1989), and so it is perhaps not surprising that there is structure in the color map, likely due to clumpiness in the dust distribution. There is good correspondence between the reddest areas in the color map and sources of $\mathrm{CO}$ emission: the central red feature in the color map corresponds to Walter et al. (2002) CO source R2, while the red area to the south is their CO source R1. The $J-K$ colors of R1 and R2 in 1 arcsec diameter apertures are $1.4(\mathrm{R} 1)$ and 1.5 (R2), while $J-K \sim 1$ in the majority of pixels near the center of NGC 3077. If dust is the main cause of the color variations in Figure 2 then regions $\mathrm{R} 1$ and $\mathrm{R} 2$ are thus subjected to reddening in $J-K$ that is $0.4-0.5$ mag greater than the main body of the galaxy. In fact, the area near R1 and R2 is the site of relatively hard x-ray emission (Ott et al. 2003), which is likely a consequence of dust absorption. Finally, while R1 appears as an elongated single source in the Walter et al. (2002) CO map, there are two distinct pockets evident in the $J-K$ map, suggesting that higher angular resolution $\mathrm{CO}$ observations will likely resolve R1 into (at least) two sources.

The $(J-H, H-K)$ two-color diagram (TCD) of individual pixels in the central $12 \times 14$ arcsec region of NGC 3077 is shown in Figure 3. The majority of pixels are concentrated near $J-H=0.55$ and $H-K=0.4$, and there is an extended plume of points that parallels the reddening vector, as expected if there are significant differences in the line of sight absorption near the center of NGC 3077. The overall length of the plume on the TCD parallel to the reddening vector suggests that differential reddening of size $\Delta \mathrm{A}_{V}=4$ magnitudes is present. Many of the pixels that are located above and to the right of the main body of points in the left hand panel of Figure 3 are in the vicinity of R1 and R2. Indeed, the colors of the $\mathrm{CO}$ source $\mathrm{R} 1$ are $J-H=0.75$ and $H-K=0.62$ within a 1 
arcsec diameter aperture, while $J-H=0.90$ and $H-K=0.57$ for CO source R2.

To aid in the interpretation of the near-infrared SED of NGC 3077, stellar sequences from Bessell \& Brett (1988) are shown in Figure 3, along with the mean colors of blue compact dwarf galaxies (BCDGs), starburst/HII galaxies and Virgo cluster dEs based on data published by Thuan (1983), Hunt, Giovanardi, \& Helou (2002), and James (1994). It is evident that the near-infrared SED of NGC 3077 differs from that of stars and most other dwarf galaxies, including those that are currently forming stars. While the majority of pixels near the center of NGC 3077 have $J-H$ colors that do not differ from those of a typical Virgo cluster $\mathrm{dE}$, these same pixels have $H-K$ colors that are $0.2-0.4$ mag redder than in a typical dE. The majority of pixels in the center of NGC 3077 also have colors that place them to the right of the BCDG and starburst/HII galaxy data on the TCD, although there is some overlap. Indeed, two of the BCDGs in the Thuan (1983) sample (Mrk 36 and Mrk 59) and two of the starburst/HII galaxies in the Hunt et al. (2002) sample (NGC 520 and NGC 695) have integrated near-infrared colors that fall near the trend defined by the NGC 3077 data in Figure 3. Simple models of the near-infrared SED of NGC 3077 are explored in the next section.

\subsection{Modelling the Near-IR SED}

As one of the nearest and best-studied dE galaxies, NGC 205 is a prime comparison object for NGC 3077, and the stellar contents of NGC 205 and NGC 3077 are similar in at least some respects. For example, there is a large population of cool AGB stars in NGC 205, indicating that there have been recent episodes of star formation (Richer, Crabtree, \& Pritchet 1984; Davidge 1992, 2003; Lee 1996; Demers, Battinelli, \& Letarte 2003). In fact, the youngest stars in NGC 205 have ages $\log \left(\mathrm{t}_{y r}\right) \sim 8.0$ (e.g. Davidge 2003; Cappellari et al. 1999), which is comparable to the time since the last interaction between NGC 3077 and M81. Moreover, the young population in NGC 3077 is concentrated within the central 700 parsecs (Thronson et al. 1991), while in NGC 205 the youngest stars are located within the central 600 parsecs (Davidge 2003). Finally, the colors of bright AGB stars in NGC 205 suggest that the intermediate age population in this galaxy formed from gas with a near-solar metallicity (Davidge 1992). While the metallicity of NGC 3077 is uncertain, the emission spectrum suggests that gas in NGC 3077 may be as metal-rich as solar (Martin 1997).

$J, H$, and $K^{\prime}$ images of NGC 205 were obtained with the CFHTIR during the June 2001 observing run, and these were processed to simulate the appearance of this galaxy if viewed at the same distance and with the same angular resolution as NGC 3077. The NGC 
205 images were reduced using the procedures described in $\S 2$, and the pixels in the results were block-averaged in $4 \times 4$ groups to simulate the loss of resolution due to the increase in distance. The block-averaged frames were then convolved with a $\sigma=0.7 \operatorname{arcsec}$ Gaussian.

The near-infrared TCD of pixels in the central $12 \times 14$ arcsec of the distance-processed NGC 205 image is shown in the right hand panel of Figure 3. The central regions of NGC 205 have an integrated SED that is similar to those of cool giants with modest amounts of reddening, and the Virgo cluster dEs studied by James (1994). For comparison, the near-infrared SED near the center of NGC 3077 is dominated by an extremely young population. To demonstrate this point, the locations of selected $\mathrm{z}=0.020, \alpha=2.35$, $\mathrm{M}_{u p}=100 \mathrm{M}_{\odot}$ instaneous burst models from Leitherer et al. (1999) are shown in Figure 3. Solar metallicity models were selected for this comparison based on the strengths of emission lines in the spectrum of NGC 3077 (Martin 1997). An extrapolation along the reddening vector from the main concentration of NGC 3077 data on the TCD passes close to the $\log \left(\mathrm{t}_{y r}\right)=6.6$ model; the $\log \left(\mathrm{t}_{y r}\right)=6.8$ and 7.0 models have $H-K$ colors that are bluer than the SED of NGC 3077. The presence of a dominant population with an age $\log \left(\mathrm{t}_{y r}\right)<7.0$ is consistent with (1) the ages of supershells in NGC 3077, which have $\log \left(\mathrm{t}_{y r}\right)$ between 6.0 and 7.0 (Ott et al. 2003), and (2) the large population of hot stars needed to produce the radio continuum flux measured by Meier, Turner, \& Beck (2001).

A system with a NGC 205-like SED can contribute only a modest fraction of the near-infrared light from the central regions of NGC 3077. To demonstrate this point, the SEDs of the $\log (\mathrm{t})=6.0$ and 6.8 models were combined with the midpoint of the NGC 205 data distribution in the TCD in varying proportions and the results are shown in Figure 4. Models in which a $\log (\mathrm{t})=6.0$ population contributes at least $2 \times$ the light from a population like that in NGC 205 in the $K$-band reproduce the unreddened near-infrared SED of NGC 3077 very well if the majority of pixels have $A_{V}$ between 3 and 4 . The models involving the $\log (\mathrm{t})=6.8 \mathrm{SED}$ give a poorer match to the NGC 3077 data for all possible contributions from a NGC 205-like SED. For example, a model made up exclusively of a population with $\log (\mathrm{t})=6.8$ differs from the main concentration of NGC 3077 data on the TCD at roughly the $1-\sigma$ level, based on the estimated uncertainty in the photometric calibration, while a model in which a NGC 205-like system contributes $10 \%$ of the near-infrared flux differs from the main concentration of NGC 3077 data at roughly the $2-\sigma$ level. 


\subsection{The Near-Infrared Isophotal Properties of NGC 3077 and NGC 205}

The $K^{\prime}$ image in the right hand panel of Figure 2 shows little structure and is radially symmetric about the bright nucleus, which is roughly 1.8 arcsec north and east of the isophotal center of the galaxy, and 1 arcsec to the north and east of the CO source R2. The general appearance of the $K^{\prime}$ data suggests that non-uniformities in the dust absorption do not greatly affect the $K$ light profile of NGC 3077. This is not unexpected, given that $\mathrm{A}_{K}=0.11 \times \mathrm{A}_{V}$ (e.g. Rieke \& Lebofsky 1985), so that the $\Delta \mathrm{A}_{V}=4$ magnitude range in

extinction, deduced from the distribution of points on the TCD in Figure 3, corresponds to only $0.4 \mathrm{mag}$ in $\mathrm{A}_{K}$.

The structural properties of the central regions of NGC 3077 at near-infrared wavelengths have been investigated using the isophote-fitting routine Ellipse (Jedrzejewski 1987), and the radial behaviours of surface brightness, ellipticity, and position angle in the final $K^{\prime}$ image are shown in Figure 5. Given that only the relatively high surface brightness central regions of the galaxy are considered it is perhaps not surprising that the estimated errors in the NGC 3077 surface brightness measurements due to uncertainties in the sky level are relatively modest, amounting to only $10 \%$ at the largest radius considered.

The $K$ surface brightness profile of NGC 3077 can be matched with either an $\mathrm{r}^{1 / 4}$ or exponential law outside of the seeing disk. The ellipticity increases, and the position angle drops, with increasing radius. Such radial changes in ellipticity and position angle are signatures of isophotal twisting, which simulations indicate could result from galaxy-galaxy interactions (e.g. Choi, Guhathakurta, \& Johnston 2002).

Colors were also computed from the isophotal measurements. $J-H$ and $H-K$ do not change with radius out to 20 arcsec from the nucleus, with $\overline{J-H}=0.55$ and $\overline{H-K}=0.4$ in this region. Uncertainties in the sky background, coupled with the extended nature of NGC 3077, prevented the measurement of reliable colors at larger radii. However, NGC 3077 is in the 2MASS Extended Source Catalogue (Jarrett et al. 2000), and the integrated colors measured from the 2MASS images are $J-H=0.58$ and $H-K=0.26$ within a radius of 109 arcsec, and $J-H=0.57$ and $H-K=0.25$ within a radius of 148 arcsec. These large radius 2MASS measurements indicate that the $J-H$ and $H-K$ colors in the outer regions of NGC 3077 are in better agreement with those of BCDGs and Virgo cluster dEs than the central regions of the galaxy, suggesting that the young stellar content in NGC 3077 is concentrated towards the center of the galaxy.

The isophotal properties of NGC 205 were measured from the distance-shifted NGC $205 K^{\prime}$ images, and the results are shown in the right hand column of Figure 5 . The $K^{\prime}$ surface brightness profile of NGC 205 can be matched with either an $\mathrm{r}^{1 / 4}$ or exponential law 
when $r>1.5$ arcsec, in agreement with what was concluded by Kent (1987) from $r$-band data. The ellipticity and position angle of NGC 205 both change with radius in Figure 5, and similar trends are seen in Kent's (1987) $r$-band data.

While the isophotal properties of NGC 205 and NGC 3077 are not identical, there are similarities. In particular, although NGC 3077 has a higher mean surface brightness than NGC 205, the central $K$ surface brightnesses of both galaxies are roughly 1 mag $\operatorname{arcsec}^{-2}$ brighter than would be inferred by extrapolating the surface brightness profile at larger radii to the galaxy center. Both galaxies also show radial changes in ellipticity and position angle. While NGC 205 shows a greater range in ellipticity than in NGC 3077, the amount of isophotal twisting, as measured from the change in position angle with radius outside of the seeing disk, is similar. Finally, after correcting for the presence of a young population, the $K$-band surface brightnesses of NGC 3077 and NGC 205 are not greatly different. In $\S 3.2$ it was demonstrated that very young stars contribute at least twice as much as a NGC 205-like system to the integrated $K$-band light from NGC 3077. If the young stars are uniformly mixed with the NGC 205-like population near the center of NGC 3077, then the surface brightness of the underlying NGC 205-like component in NGC 3077 will be at least 1.2 magnitudes fainter in $K$ than in the composite system. Shifting the $K$-band surface brightness profile of NGC 3077 fainter by this amount, and brightening the results by 0.3 - 0.4 magnitudes to correct for dust absorption in NGC 3077, yields a $K$-band surface brightness profile that is in much better agreement with that observed for NGC 205 than the original NGC 3077 profile.

\section{STAR CLUSTERS IN NGC 3077}

Studies of star clusters in NGC 3077 will provide insight into the evolution of the galaxy. The approximate faint limit of the current data is $K=18$, and there are a number of sources brighter than this in the CFHTIR field that may be clusters belonging to NGC 3077. The locations, brightnesses, and colors of sources with $K \leq 18$ are summarized in Table 1. Crowding makes it difficult to resolve individual sources in the central 15 arcsec of NGC 3077 and, while objects with $K \leq 18$ are present near the center of the galaxy, there is a chance that these may be blends of bright stars or clusters. Therefore, only objects with $K<16$ within 15 arcsec of the nucleus are listed in Table 1.

The $x, y$ co-ordinates in Table 1 are in pixel units, as measured by the DAOPHOT FIND routine. The origin is in the lower left hand corner of Figure 1, while the upper right hand corner is $(x, y)=(1000,1000)$. The columns labelled $\Delta \delta$ and $\Delta \alpha$ give offsets, in arcsec, from the isophotal center of NGC 3077 along the declination and right ascension axes, with 
positive offsets to the north and west. The CFHTIR science field was not perfectly aligned along the cardinal axes, and the entries in the fourth and fifth columns of Table 1 have been adjusted for this. A scale of 0.211 arcsec pixel ${ }^{-1}$ was adopted when computing these offsets.

Some of the sources listed in Table 1 are in the 2MASS Point Source Catalogue (Cutri et al. 2003), and hence can be used to check the photometric calibration. There are 5 sources in common with the 2MASS survey that are brighter than $K=16$ and are located more than 0.5 arcminutes from the center of NGC 3077. A comparison between the CFHTIR and 2MASS photometry gives mean differences, in the sense Davidge - 2MASS, of $\Delta K=0.03 \pm 0.03, \Delta(J-H)=0.05 \pm 0.07$, and $\Delta(H-K)=0.01 \pm 0.04$, where the quoted errors are the standard deviations about the mean difference. These mean differences are consistent with the uncertainties in the photometric calibration estimated from standard star measurements $(\S 2)$.

The objects in Table 1 have been classified as either foreground stars (FS), old globular clusters (OGC), or young clusters (YC) based on their near-infrared photometric properties. The near-infrared CMDs and TCD of these objects are shown in Figures 6 and 7; objects identified as foreground stars are not shown in Figure 7 to prevent cluttering this figure. The criteria used to make the identifications are discussed in the following sections.

\subsection{Foreground Stars}

Likely foreground stars can be identified using near-infrared colors and brightnesses. The majority of foreground stars should be on the lower main sequence and, unless they have very late spectral-types, will have $H-K<0.3$, and $J-H<0.7$ (e.g. Bessell \& Brett 1988). Foreground stars will also have stellar SEDs and, since NGC 3077 is at a Galactic latitude $b \sim 40^{\circ}$, will likely have only modest amounts of reddening. Finally, some foreground stars may be too bright to be plausible members of NGC 3077.

There are eight sources with $K<16.8$ that form a well-defined sequence in the CMDs in Figure 6, with $\overline{J-K}=0.9$ and $\overline{H-K}=0.2$. While not plotted in Figure 7 , these objects also fall close to the stellar sequences on the TCD. Hence, these objects are identified in Table 1 as foreground stars.

\subsection{Globular Clusters and the Specific Cluster Frequency}

Harris \& van den Bergh (1981) defined the specific frequency of globular clusters, $S_{N}$, as the number of clusters per unit galaxy luminosity, normalized to $\mathrm{M}_{B}=-15$. Subsequent 
studies have demonstrated that there are systematic galaxy-to-galaxy differences in $S_{N}$, that are related, at least in part, to the structural properties of the host galaxy. In particular, $\mathrm{S}_{N}$ in disk-dominated Local Group galaxies is an order of magnitude lower than in spheroidal systems (e.g. van den Bergh 1995). The Local Group dEs NGC 147, 185, and 205 have $\overline{S_{N}}=4.3 \pm 1.2$ (Harris 1991), which is similar to $\mathrm{S}_{N}$ measured in Virgo and Fornax cluster dE's (Durrell et al. 1996; Miller et al. 1998), while Local Group dIrr galaxies have markedly lower $\mathrm{S}_{N}$ 's, with $\mathrm{S}_{N}=0.4-0.5$ in the SMC and LMC (Harris 1991). Differences of this nature may be due to the properties of the fragments from which the galaxies were assembled (Harris \& Harris 2002).

The globular cluster content of NGC 3077 may provide clues about the nature of the galaxy prior to the recent interactions. If NGC 3077 was originally a dE galaxy and has not lost a significant number of clusters due to tidal stripping, then it should have an entourage of some 30 globular clusters if $\mathrm{M}_{B}=-17$ (Walter et al. 2002) and $\mathrm{S}_{N}=4$. On the other hand, if NGC 3077 were initially a dIrr with $\mathrm{S}_{N}=0.5$ then there should be at most $3-4$ clusters.

The typical brightness of globular clusters in NGC 3077 can be estimated from the cluster content of other nearby galaxies. The peak luminosity of the globular cluster luminosity function (GCLF) remains constant to within a few tenths of a magnitude both in (e.g. Kavelaars \& Hanes 1997) and between (e.g. Kundu \& Whitmore 2001; Larsen et al. 2001; Harris 1991) galaxies. The M31 cluster system is the best studied extragalactic system, and the apparent magnitudes expected for globular clusters in NGC 3077 can be estimated from the M31 cluster data. The brightest globular cluster in M31, 023-078, has $K=10.7$ (Barmby et al. 2000), which corresponds to $\mathrm{M}_{K}=-13.8$, or $K=14$ at the distance of NGC 3077. Hence, objects brighter than $K=14$ near NGC 3077 are almost certainly not globular clusters. The brightest globular cluster in NGC 3077 will likely be much fainter than 023-078, as NGC 3077 is much less massive than M31.

The peak of the M31 GCLF occurs near $\mathrm{M}_{K}=-10$ (Barmby, Huchra, \& Brodie 2001), which corresponds roughly to $K=18$ in NGC 3077. Consequently, globular clusters in NGC 3077 should occur in ever increasing numbers near the faint limit of the CFHTIR data. In fact, a population of objects with a broad range of colors is evident in the CMDs in Figure 6 when $K>16.8$, and when placed on the TCD these objects have SEDs that are similar to those of classical globular clusters in M31, with only modest amounts of dust absorption. Twelve sources are identified as classical globular clusters in this way in Table 1. Some of these will almost certainly turn out to be foreground stars. Indeed, based on number counts between $K=16$ and 17 , it can be anticipated that $3-4$ of the suspected classical globular clusters with $K$ between 17 and 18 are foreground stars. 
The candidate globular clusters in Table 1 sample only the upper half of the expected range of cluster brightnesses, and so a number of fainter globular cluster candidates wait

to be discovered. If, as suggested by number counts of foreground stars with $K$ between 16 and 17, 8 of the candidate clusters are confirmed as belonging to NGC 3077, and half of the cluster content has yet to be detected because it is below the detection limit of the CFHTIR data, which is near the approximate mid-point of the globular cluster luminosity distribution, then $\mathrm{S}_{N}=2.5$ in NGC 3077. For comparison, $\mathrm{S}_{N}=2.3 \pm 0.3$ in NGC 205 (Harris 1991). The specific frequency of globular clusters in NGC 3077 thus appears to overlap with that observed in dE galaxies.

\subsection{Young Clusters}

The formation of compact star clusters is associated with large-scale star-forming episodes (e.g. Conti \& Vacca 1994; Whitmore et al. 1999), when massive molecular clouds are expected to be present (e.g. Harris \& Pudritz 1994). Given the level of star-forming activity, Chandar et al. (2001) suggest that massive young star clusters might be present in NGC 3077. If the clusters are sufficiently young, then they will have SEDs that differ markedly from those of old globular clusters, and so can be identified with the current data. In fact, the models of young populations from Leitherer et al. (1999), shown in Figure 3, predict that clusters with ages $\log \left(\mathrm{t}_{y r}\right)<7$ have much redder $H-K$ colors than those of globular clusters, due to the contribution made by ionized gas to the SED of young systems. Fourteen objects with very red colors are seen in the CMDs in Figure 6, and these are identified as compact young clusters in Table 1.

When placed on the TCD in Figure 7, the candidate young clusters have SEDs that are similar to those of individual pixels near the center of NGC 3077. In addition, the suspected young clusters form a sequence in the TCD that parallels the reddening vector, indicating that dust is likely the dominant source of dispersion in the near-infrared SEDs of these objects. Comparisons with the Leitherer et al. (1999) models in Figure 7 suggest that the candidate young clusters have ages $\log \left(\mathrm{t}_{y r}\right)$ between 6.0 and 6.6 , which is comparable to the ages of gas shells in NGC 3077 (Ott et al. 2003).

The line of sight extinction for each candidate young cluster was estimated by extrapolating along the reddening vector to a point midway between the $\log \left(\mathrm{t}_{y r}\right)=6.0$ and 6.6 models in Figure 7, and the results, along with the absolute brightness of each source, are summarized in Table 2. The brightest blue supergiants in galaxies have $\mathrm{M}_{K}=-10$ (Rozanski \& Rowan-Robinson 1994), and the suspected young clusters identified here are brighter than this; hence, the sources listed in Table 2 are too bright to be individual 
massive stars.

The masses of the suspected young clusters were estimated using the $\mathrm{M}_{K}$ values in Table 2 and the integrated $K$-band brightnesses predicted by the $\mathrm{z}=0.020$ Leitherer et al. (1999) models with $\alpha=2.35, \mathrm{M}_{\text {low }}=1 \mathrm{M}_{\odot}$, and $\mathrm{M}_{u p}=100 \mathrm{M}_{\odot}$. At a fixed mass, the integrated brightness of young systems is age-sensitive, and so masses were computed for assumed ages $\log \left(\mathrm{t}_{y r}\right)=6.0$ and 6.6. The results are shown in the last two columns of Table 2. The masses computed in this way are not sensitive to the adopted metallicity, but they do depend on the nature of the assumed initial mass function. For example, masses computed using the $\mathrm{M}_{u p}=30 \mathrm{M}_{\odot}$ Leitherer et al. (1999) models would be 0.4 dex higher than those listed in Table 2. The mass estimates are also sensitive to the adopted low mass cut-off, and models that assume $\mathrm{M}_{\text {low }}<1 \mathrm{M}_{\odot}$ will give higher cluster masses than those listed in Table 2. Finally, some of the $K$-band light may also come from thermal emission from hot dust, which may occur in dense concentrations of hot stars (e.g. Hunt et al. 2002), and emission of this nature will cause the masses in Table 2 to be over-estimated. There is also a bias towards detecting clusters with thermal dust emission, as these objects will be brighter at $2 \mu \mathrm{m}$ than clusters with similar mass but lacking this light component.

The majority of suspected young clusters have masses between $\log \left(\mathrm{M}_{\odot}\right)=4$ and 5 , with a mean $\log \left(\mathrm{M}_{\odot}\right)=4.5$ if $\log \left(\mathrm{t}_{y r}\right)=6.0$, and 4.8 if $\log \left(\mathrm{t}_{y r}\right)=6.6$. The uncertainties discussed in the preceeding paragraph notwithstanding, the masses derived for the candidate young clusters in NGC 3077 are in good agreement with those seen in other star-forming regions, such as the circumnuclear environment of M83 (Harris et al. 2001). The most massive cluster is located close to the isophotal center of the galaxy. The nuclei of nucleated $\mathrm{dE}(\mathrm{dE}, \mathrm{n})$ galaxies have globular cluster-like masses (e.g. Binggeli \& Cameron 1991), and the masses estimated for the nuclear cluster in NGC 3077 in Table $2(\log M=5.8$ and 6.1 for $\log \left(\mathrm{t}_{y r}\right)=6.0$ and 6.6) fall within the mass range of Galactic globular clusters (Pryor \& Meylan 1993).

\section{DISCUSSION AND SUMMARY}

\subsection{The Near-Infrared SED of NGC 3077}

Sub-arcsec angular resolution $J, H$, and $K^{\prime}$ images obtained with the CFHTIR camera

have been used to investigate the near-infrared photometric properties of the central regions of the M81 group galaxy NGC 3077. In $\S 3$ it was demonstrated that (1) the integrated near-infrared SED of the central regions of NGC 3077 differs from that of 'typical' dE's, BCDG's and HII/starburst galaxies, and (2) the light near $2 \mu \mathrm{m}$ is dominated by very 
young stars. The latter result is perhaps not surprising, as previous studies have found that the SFR in NGC 3077 is high, although there is significant scatter among the estimates. Thronson et al. (1991) estimate that the SFR is $0.06 \mathrm{M}_{\odot}$ year $^{-1}$ based on the integrated $\mathrm{H} \alpha$ flux, and $\leq 0.25 \mathrm{M}_{\odot}$ year $^{-1}$ from FIR emission. Walter et al. (2002) compute a SFR of $0.05 \mathrm{M}_{\odot}$ year $^{-1}$ based on extinction-corrected $\mathrm{H} \alpha$ measurements. Meier et al. (2001) estimate that the SFR is $0.4 \mathrm{M}_{\odot}$ year $^{-1}$ from the $2.6 \mathrm{~mm}$ continuum flux, while Ott et al. (2003) estimate that the SFR is $0.6 \mathrm{M}_{\odot}$ year ${ }^{-1}$ based on the energy needed to create super gas shells. As noted by Ott et al. (2003), the high SFR inferred in their study may suggest that the SFR has dropped recently. While the present-day SFR in NGC 3077 is markedly lower than in M82 (e.g. Ott et al. 2003), the efficiency with which stars form out of molecular gas in both galaxies is similar (Walter et al. 2002).

The detection of a population of suspected young star clusters, which have ages $\log \left(\mathrm{t}_{y r}\right)$ between 6.0 and 6.8 and masses $\log \left(\mathrm{M}_{\odot}\right)$ between 4.0 and $5.0(\S 4)$, is consistent with a very high recent SFR. The total integrated mass in the young clusters is $1-2 \times 10^{6} \mathrm{M}_{\odot}$. Given that the near-infrared SEDs of these clusters are suggestive of an age $\log \left(\mathrm{t}_{y r}\right) \leq 6.6$, then the SFR needed to produce these objects is $0.25-0.50 \mathrm{M}_{\odot}$ year ${ }^{-1}$, which falls within the range of estimates computed using other techniques. This is a lower limit to the total SFR, as it does not include stars that formed in clusters that have been disrupted, or clusters that are below the faint limit of these data.

The near-infrared SED of NGC 3077 does not preclude a modest contribution from a system having an SED like that of NGC 205. The presence of such a population is consistent with the detection of RGB stars in NGC 3077 by Sakai \& Madore (2001) and Karachentsev et al. (2002), and the discovery of a healthy number of candidate old globular clusters ( $\S 4)$. Thus, NGC 3077 is not a recently formed system, as may be the case for some members of the M81 group (Yun et al. 1994, Boyce et al. 2001).

The models used here to simulate the near-infrared SED of NGC 3077 include contributions from stars and gas emission. However, Hunt et al. (2002) investigated the infrared photometric properties of star-forming galaxies, and concluded that thermal emission from hot dust may contribute significantly to the light from very active star-forming systems at wavelengths longward of $2 \mu \mathrm{m}$, and there are hints that emission from hot dust may contribute significantly to the infrared light from NGC 3077. One clue comes from the near-infrared SED. Thermal emission from dust with temperatures cooler than a few hundred $\mathrm{K}$ does not contribute significantly to light at wavelengths shortward of $2 \mu \mathrm{m}$, and so the $J-H$ color of a system with significant dust emission will be the same as from a galaxy lacking this emission, while the $H-K$ color may be much redder. It is evident from Figure 3 that the majority of pixels near the center of NGC 3077 have $J-H$ colors that 
are consistent with those of dEs and BCDGs, but have $H-K$ colors that are redder than in these systems, as expected if emission from hot dust is present. Emission from hot dust will likely be concentrated in the dense central star-forming regions of NGC 3077, where the radiation field is most intense. In $\S 3.3$ it was shown that the $J-H$ color measured near the center of NGC 3077 is similar to that measured at larger radii from 2MASS survey data, while the central $H-K$ color is much redder than at large radii, and so the radial color profile is also consistent with emission from hot dust. Finally, when compared with other galaxies, NGC 3077 has a very low mass of cool gas (Stickel et al. 2000), as might be expected if a large fraction of the dust is heated by young stars. Observations of NGC 3077 in the $3-5 \mu \mathrm{m}$ region will allow firmer constraints to be placed on any contribution made by thermal emission from hot dust. It is evident from Figure 4 that if significant emission from hot dust is present in NGC 3077 then the net result will be to allow for a larger contribution from a NGC 205-like SED.

\subsection{The Evolution of NGC 3077}

Tidal interactions are common events in the local Universe, as there are debris trails in the halos of the Milky-Way (e.g. Ibata et al. 1995), M31 (e.g. Ibata et al. 2001), and in nearby groups (e.g. Boyce et al. 2001). Recognizing that tidal interactions can affect the structural characteristics of galaxies, and can also explain the morphology-density relation between dIrr and dSph systems, Mayer et al. (2001) suggested that the dSph and dE companions of the Milky-Way and M31 may have originally been gas-rich disky galaxies that were transformed by tidal interactions into spheroidal systems. Mayer et al. (2001) suggest that tides trigger bar instabilities that channel gas into the central regions of the progenitor, where star formation occurs. Feedback then heats the ISM, while the bar re-distributes angular momentum to the outer regions of the galaxy, which are subsequently stripped away. The bar eventually buckles, and the result is a system that has lost much of its angular momentum and has been transformed from a gas-rich disky to a gas-poor spheroidal morphology. Whether the final product is a $\mathrm{dSph}$ or a $\mathrm{dE}$ depends on the surface brightness of the progenitor, in the sense that dSphs evolve from low surface brightness dIrrs that experience multiple bursts of star formation, while dEs evolve from higher surface brightness dIrrs that experience one dominant, extended, star-forming episode that occurs over a $1-2$ Gyr period. Simulations indicate that the time scale for the transformation is a few Gyr, so there is a reasonable expectation of viewing this process at work in nearby galaxy groups.

While tidal interactions undoubtedly influence galaxy evolution, they do not provide a 
panacea for explaining dwarf galaxy morphology. Indeed, there are isolated dSph galaxies, such as Tucana, that likely have not been subjected to tidal interactions but still show remarkable similarities to dSphs in denser environments. The bar-driven transformation process described by Mayer et al. (2001) should have a major impact on radial population gradients, and yet the radial population behaviour of Tucana is similar to dSphs in denser environments (Harbeck et al. 2001). There are also galaxies in hierarchical systems that appear not to have been altered by tidal forces. In particular, the surface brightness profile and central black hole mass of M32, which is a galaxy that many have argued may be an extreme endpoint of tidal pruning (e.g. Faber 1973; Nieto 1990), suggest that the structural characteristics of this galaxy were imprinted early on and have not since been greatly altered (Graham 2002).

Tidal interactions are clearly affecting the properties of NGC 3077. Much of the atomic gas associated with NGC 3077 is in a tidal arm that is well offset from the main body of the galaxy (Walter et al. 2002; Yun et al. 1994), while there is a string of molecular complexes extending to the north and west of the center of NGC 3077 (Meier et al. 2001), the positioning of which may also be the result of tidal effects. While the interstellar medium of NGC 3077 is clearly being disrupted, when integrated over a large area, the HI mass to light ratio of NGC 3077 is more appropriate for a dIrr, rather than a dE (Walter et al. 2002), as might be expected if NGC 3077 is undergoing a morphological transformation. Of course, it is possible that some of the gas currently associated with NGC 3077 may have been stripped from another galaxy.

Simulations discussed by Mayer et al. (2001) suggest that the time scale for bar evolution in tidally influenced gas-rich dwarf galaxies is on the order of a few Gyr, and so there is a reasonable expectation of observing barred tidally interacting dwarf galaxies. In fact, stars in the bar of the LMC have an age of $\sim 5$ Gyr (Smecker-Hane et al. 2002), supporting the notion that the bars in dIrr galaxies in hierarchical systems can be stable against buckling for long periods of time. The timescale for bar disruption is much longer than the time since the last major encounter between M81, M82, and NGC 3077, so if a bar formed in NGC 3077 after the last encounter then it should still be present. This being said, the near-infrared images of NGC 3077 do not show evidence of a bar, and the peaky light profile of NGC 3077 (§3.3) is not consistent with a bar-dominated light distribution.

dIrr and $\mathrm{dE}$ galaxies have very different $\mathrm{S}_{N}$ 's (e.g. Harris 1991), and so the old globular cluster content of NGC 3077 may provide clues about the nature of the galaxy prior to its most recent encounter with M81. In particular, if NGC 3077 were a 'typical' $\mathrm{dE}$ before encountering M81 then it should contain a rich population of classical globular clusters, while if it were recently a dIrr galaxy then it should contain only a modest 
population of such objects. A caveat is that the young clusters that form during vigorous star-forming episodes can have globular cluster-like masses, as appears to be the case in NGC 3077 (§4.3). If these clusters are not disrupted then, when viewed in a few Gyr, the galaxy will contain an even larger globular cluster population, albeit spanning a range of ages. The $\mathrm{S}_{N}$ may thus change with time over the course of the transformation process.

Spectroscopic observations, which will yield line strengths and radial velocities, will be essential to distinguish between actual clusters and faint field stars in NGC 3077. The globular clusters in NGC 3077 will likely be more metal-poor than the surrounding field population (e.g. da Costa \& Mould 1988), and so will likely have weak absorption lines. Higher angular resolution images will also be useful for identifying clusters, as both globular clusters (Kundu \& Whitmore 2001) and compact young clusters (Chandar et al. 2001; Harris et al. 2001) have characteristic sizes of a few parsecs, and so will have a non-stellar appearance when viewed with image qualities approaching 0.1 arcsec FWHM.

Although lacking spectra and high-resolution images, the present data still provide tantalizing hints into the nature of NGC 3077. In $\S 4.2$ it was estimated that $\mathrm{S}_{N}=2.5$, suggesting that the specific frequency of globular clusters in NGC 3077 is similar to that of NGC 205. The specific globular cluster frequency is consistent with the structural characteristics of NGC 3077, which suggest that if star formation was terminated immediately then the galaxy would fade to become a dE,n. While the young central cluster in NGC 3077 is offset slightly from the isophotal center of the galaxy, this occurs in roughly $20 \%$ of dE,n (e.g. Binggeli, Barazza, \& Jerjen 2000).

Thanks are extended to the referee, Leslie Hunt, for providing comments that greatly improved the manuscript. 


\begin{tabular}{|c|c|c|c|c|c|c|c|c|}
\hline ID \# & $\begin{array}{r}x \\
\text { (pixels) }\end{array}$ & $\begin{array}{r}y \\
\text { (pixels) }\end{array}$ & $\begin{array}{c}\Delta \alpha \\
(\operatorname{arcsec})\end{array}$ & $\begin{array}{c}\Delta \delta \\
(\operatorname{arcsec})\end{array}$ & $\bar{K}$ & $J-H$ & $H-K$ & Object Type $^{a}$ \\
\hline 1 & 31.5 & 461.8 & -100.1 & 0.0 & 17.987 & 0.689 & -0.018 & $\mathrm{OGC}$ \\
\hline 2 & 58.2 & 751.5 & -99.0 & +61.0 & 15.666 & 0.513 & 0.407 & $\mathrm{FS}$ \\
\hline 3 & 81.3 & 490.8 & -90.1 & +6.3 & 17.453 & 0.753 & 0.704 & $\mathrm{YC}$ \\
\hline 4 & 89.0 & 294.7 & -85.5 & -34.6 & 17.045 & 0.524 & 0.221 & $\mathrm{OGC}$ \\
\hline 5 & 122.7 & 674.8 & -84.2 & +45.8 & 15.027 & 0.612 & 0.272 & FS \\
\hline 6 & 154.3 & 239.2 & -70.9 & -45.4 & 16.709 & 0.992 & 0.794 & $\mathrm{YC}$ \\
\hline 7 & 157.4 & 140.4 & -68.7 & -66.1 & 16.901 & 0.756 & 0.662 & $\mathrm{YC}$ \\
\hline 8 & 180.8 & 786.2 & -73.7 & +70.2 & 16.676 & 0.647 & 0.206 & FS \\
\hline 9 & 229.8 & 534.8 & -59.5 & +18.0 & 16.993 & 0.867 & 0.643 & $\mathrm{YC}$ \\
\hline 10 & 281.0 & 212.0 & -43.8 & -49.1 & 16.608 & 0.693 & 0.629 & $\mathrm{YC}$ \\
\hline 11 & 282.1 & 555.8 & -48.8 & +23.3 & 15.636 & 0.562 & 0.231 & $\mathrm{FS}$ \\
\hline 12 & 290.4 & 564.2 & -47.2 & +25.2 & 16.670 & 0.679 & 0.646 & $\mathrm{YC}$ \\
\hline 13 & 310.9 & 312.7 & -39.0 & -27.5 & 17.755 & 0.933 & 0.411 & $\mathrm{OGC}$ \\
\hline 14 & 315.9 & 46.0 & -33.8 & -83.5 & 17.852 & 0.496 & 0.583 & $\mathrm{YC}$ \\
\hline 15 & 371.0 & 693.4 & -32.3 & +53.6 & 15.981 & 0.601 & 0.188 & FS \\
\hline 16 & 400.3 & 426.1 & -22.0 & -2.2 & 17.435 & 0.750 & 0.380 & $\mathrm{OGC}$ \\
\hline 17 & 411.7 & 443.2 & -19.8 & +1.6 & 16.871 & 0.776 & 0.308 & $\mathrm{OGC}$ \\
\hline 18 & 414.7 & 551.2 & -20.9 & +24.3 & 14.530 & 0.563 & 0.202 & FS \\
\hline 19 & 438.3 & 349.3 & -12.8 & -17.8 & 17.197 & 0.938 & 0.793 & $\mathrm{YC}$ \\
\hline 20 & 497.2 & 429.9 & -1.6 & 0.0 & 15.840 & 1.214 & 0.936 & $\mathrm{YC}$ \\
\hline 21 & 503.9 & 429.0 & 0.0 & 0.0 & 13.213 & 0.913 & 0.613 & $\mathrm{YC}$ \\
\hline 22 & 509.5 & 416.4 & +1.2 & -2.6 & 15.698 & 0.918 & 0.629 & $\mathrm{YC}$ \\
\hline 23 & 531.3 & 399.1 & +6.0 & -5.9 & 15.640 & 0.740 & 0.395 & $\mathrm{YC}$ \\
\hline 24 & 616.6 & 418.5 & +23.7 & -0.5 & 17.459 & 0.577 & 0.164 & $\mathrm{OGC}$ \\
\hline 25 & 669.1 & 701.1 & +30.3 & +59.8 & 12.575 & 0.644 & 0.237 & FS \\
\hline 26 & 761.8 & 940.1 & +46.2 & +111.5 & 16.476 & 0.605 & 0.222 & $\mathrm{FS}$ \\
\hline 27 & 763.1 & 585.6 & +51.9 & +36.9 & 16.986 & 0.582 & 0.135 & $\mathrm{OGC}$ \\
\hline 28 & 763.4 & 291.0 & +56.5 & -25.0 & 17.766 & 0.946 & 0.392 & $\mathrm{OGC}$ \\
\hline 29 & 771.8 & 347.2 & +57.4 & -13.1 & 17.098 & 0.575 & 0.173 & $\mathrm{OGC}$ \\
\hline 30 & 869.7 & 749.6 & +71.8 & +73.1 & 17.256 & 0.334 & 0.084 & $\mathrm{OGC}$ \\
\hline 31 & 908.2 & 779.4 & +79.4 & +80.0 & 17.488 & 0.685 & 0.198 & $\mathrm{OGC}$ \\
\hline 32 & 952.4 & 862.6 & +87.5 & +98.2 & 16.820 & 0.725 & 0.253 & $\mathrm{OGC}$ \\
\hline 33 & 964.2 & 157.7 & +100.8 & -50.0 & 17.042 & 0.864 & 0.636 & YC \\
\hline
\end{tabular}

Table 1: Objects With $\mathrm{K} \leq 18$

${ }^{a} \mathrm{FS}=$ Foreground star; OGC $=$ Old globular cluster $; \mathrm{YC}=$ Young cluster 


\begin{tabular}{rcccc}
\hline \hline ID \# & $\mathrm{A}_{V}$ & $\mathrm{M}_{K}^{\text {Stellar }}$ & $\begin{array}{c}\log (\text { Mass }) \\
(\log =6.0)\end{array}$ & $\begin{array}{c}\log (\text { Mass }) \\
(\log \mathrm{t}=6.6)\end{array}$ \\
\hline 3 & 7.0 & -11.2 & 4.1 & 4.4 \\
6 & 9.1 & -12.2 & 4.5 & 4.8 \\
7 & 6.8 & -11.7 & 4.3 & 4.6 \\
9 & 7.4 & -11.7 & 4.3 & 4.6 \\
10 & 6.2 & -12.0 & 4.4 & 4.7 \\
12 & 6.2 & -11.9 & 4.3 & 4.6 \\
14 & 4.6 & -10.6 & 3.8 & 4.1 \\
19 & 8.7 & -11.7 & 4.3 & 4.6 \\
20 & 11.3 & -13.3 & 4.9 & 5.2 \\
21 & 7.7 & -15.5 & 5.8 & 6.1 \\
22 & 7.8 & -13.1 & 4.8 & 5.1 \\
23 & 5.5 & -12.9 & 4.7 & 5.0 \\
33 & 7.4 & -11.7 & 4.3 & 4.6 \\
\hline
\end{tabular}

Table 2: Intrinsic Parameters of the Candidate Young Clusters 


\section{REFERENCES}

Barbieri, C., Bertola, F., \& di Tullio, G. 1974, A\&A, 35, 463

Barmby, P., Huchra, J. P., Brodie, J. P., Forbes, D. A., Schroder, L. L., \& Grillmair, C. J. 2000, AJ, 119, 727

Barmby, P., Huchra, J. P., \& Brodie, J. P. 2001, AJ, 121, 1482

Benacchio, L., \& Galletta, G. 1981, ApJ, 243, L65

Bessell, M. S., \& Brett, J. M. 1988, PASP, 100, 1134

Bingelli, B., \& Cameron, L. M. 1991, A\&A, 252, 27

Binggeli, B., Barazza, F., \& Jerjen, H. 2000, A\&A, 359, 447

Blitz, L., \& Robishaw, T. 2000, ApJ, 541, 675

Boyce, P. J. et al. 2001, ApJ, 560, L127

Cappellari, M., Bertola, F., Burstein, D., Buson, L. M., Greggio, L., \& Renzini, A. 1999, ApJ, 515, L17

Carraro, G., Chiosi, C., Girardi, L., \& Lia, C. 2001, MNRAS, 327, 69

Chandar, R., Tsvetanov, Z., \& Ford, H. C. 2001, AJ, 122, 1342

Choi, P. I., Guhathakurta, P., \& Johnston, K. V. 2002, AJ, 124, 310

Conti, P. S., \& Vacca, W. D. 1994, ApJ, 423, L97

Cox, A. N. 2000, Astrophysical Quantities (Fourth Edition), pp. 341

Cutri, R. M. et al. 2003, 2MASS All-Sky Catalogue of Point Sources, University of Massachusetts and the Infrared Processing and Analysis Center/California Institute of Technology

da Costa, G. S., \& Mould, J. R. 1988, ApJ, 334, 159

Davidge, T. J. 1992, ApJ, 397, 457

Davidge, T. J. 2003, ApJ, 597, 289

Dekel, A., \& Silk, J. 1986, ApJ, 303, 39

Demers, S., Battinelli, P., \& Letarte, B. 2003, AJ, 125, 3037

Durrell, P. R., Harris, W. E., Geisler, D., \& Pudritz, R. E. 1996, AJ, 112, 972

Faber, S. M. 1973, ApJ, 179, 423

Ferrara, A., \& Tolstoy, E. 2000, MNRAS, 313, 291

Graham, A. W. 2002, ApJ, 568, L13 
Harbeck, D. et al. 2001, AJ, 122, 3092

Harris, W. E. 1991, ARA\&A, 29, 543

Harris, W. E., \& Harris, G. L. H. 2002, AJ, 123, 3108

Harris, W. E., \& Pudritz, R. E. 1994, ApJ, 429, 177

Harris, W. E., \& van den Bergh, S. 1981, AJ, 86, 1627

Harris, J., Calzetti, D., Gallagher, J. S., Conselice, C. J., \& Smith, D. A. 2001, AJ, 122, 3046

Hawarden, T. G., Leggett, S. K., Letawsky, M. B., Ballantyne, D. R., \& Casali, M. M. 2001, MNRAS, 325, 563

Hunt, L. K., Giovanardi, C., \& Helou, G. 2002, A\&A, 394, 873

Ibata, R. A., Gilmore, G., \& Irwin, M. J. 1995, MNRAS, 277, 781

Ibata, R., Irwin, M., Lewis, G., Ferguson, A. M. N., \& Tanvir, N. 2001, Nature, 412, 49

James, P. A. 1994, MNRAS, 269, 176

Jedrzejewski, R. I. 1987, MNRAS, 226, 747

Jarrett, T. H., Chester, T., Cutri, R., Schneider, S., Skrutskie, M., \& Huchra, J. P. 2000, AJ, 119, 2498

Karachentsev, I. D., Karachentseva, V. E., \& Boerngen, F. 1985, MNRAS, 217, 731

Karachentsev, I. D. et al. 2002, A\&A, 383, 125

Kavelaars, JJ, \& Hanes, D. A. 1997, MNRAS, 285, L31

Kent, S. M. 1987, AJ, 94, 306

Kochanek, C. S. 1996, ApJ, 457, 228

Kundu, A., \& Whitmore, B. C. 2001, AJ, 121, 2950

Larsen, S. S., Brodie, J. P., Huchra, J. P., Forbes, D. A., \& Grillmair, C. J. 2001, AJ, 121, 2974

Lee, M. G. 1996, AJ, 112, 1438

Leitherer, C. et al. 1999, ApJS, 123, 3

Lin, D. N. C., \& Faber, S. M. 1983, ApJ, 266, L21

Martin, C. L. 1997, ApJ, 491, 561

Martin, C. L. 1998, ApJ, 506, 222

Mayer, L. et al. 2001, ApJ, 559, 754 
Meier, D. S., Turner, J. L., \& Beck, S. C. 2001, AJ, 122, 1770

Miller, B. W., Lotz, J. M., Ferguson, H. C., Stiavelli, M., \& Whitmore, B. C. 1998, ApJ, 508, L133

Nieto, J-L 1990 in Dynamics and Interaction of Galaxies, R. Wielen ed. (Springer: Berlin), p. 258

Ott, J., Martin, C. L., \& Walter, F. 2003, ApJ, 594, 776

Perrett, K. M., Bridges, T. J., Hanes, D. A., Irwin, M. J., Brodie, J. P., Carter, D., Huchra, J. P., \& Watson, F. G. 2002, 123, 2490

Prada, F., \& Burkert, A. 2002, ApJ, 564, L73

Price, J. S., \& Gullixson, C. A. 1989, ApJ, 337, 658

Pryor, C., \& Meylan, G. 1993, in ASP Conf. Ser 50, Structure and Dynamics of Globular Clusters, ed. S. G. Djorgovski \& G. Meylan (San Francisco:ASP), 357

Richer, H. B., Crabtree, D. R., \& Pritchet, C. J. 1984, ApJ, 287, 138

Rieke, G. H., \& Lebofsky, M. J. 1985, ApJ, 288, 618

Rozanski, R., \& Rowan-Robinson, M. 1994, MNRAS, 271, 530

Sakai, S., \& Madore, B. F. 2001, ApJ, 555, 280

Schroder, L. L., Brodie, J. P., Kissler-Patig, M., Huchra, J. P., \& Phillips, A. C. 2002, AJ, 123,2473

Silk, J., Wyse, R. F. G., \& Shields, G. A. 1987, ApJ, 322, L59

Smecker-Hane, T. A., Cole, A. A., Gallagher, J. S. III, \& Stetson, P. B. 2002, ApJ, 566, 239

Stickel, M. et al. 2000, A\&A, 359, 865

Tajiri, Y. Y., \& Kamaya, H. 2002, A\&A, 389, 367

Thronson, H. A., Wilton, C., \& Ksir, A. 1991, MNRAS, 252, 543

Thuan, T. X. 1983, ApJ, 268, 667

Thuan, T. X. 1985, ApJ, 299, 881

van den Bergh, S. 1995, AJ, 110, 2700

van der Hulst, J. M. 1979, A\&A, 75, 97

Walter, F., Weiss, A., Martin, C., \& Scoville, N. 2002, AJ, 123, 225

Whitmore, B. C., Zhang, Q., Leitherer, C., Fall, S. M., Schweizer, F., \& Miller, B. W. 1999, AJ, 118, 1551 
Yun, M. S., Ho, P. T. P., \& Lo, K. Y. 1994, Nature, 372, 530 


\section{FIGURE CAPTIONS}

Fig. 1. - The $3.3 \times 3.3 \operatorname{arcmin} K^{\prime}$ image of NGC 3077. The diffuse light from the main body of the galaxy, a template for which was constructed by median-filtering the final processed $K^{\prime}$ image, has been subtracted out to allow sources within a few tens of arcsec of the nucleus to be seen. North is at the top, and east is to the left. The image quality is FWHM = 0.7 arcsec. The contours show the $K=20$ and 21 mag $\operatorname{arcsec}^{-2}$ isophotes, measured from the final processed $K^{\prime}$ image. The sources outside of the central regions of NGC 3077 are foreground stars and possible star clusters, and these are discussed in $\S 4$.

Fig. 2. - The central $12 \times 14$ arcsec of NGC 3077 as imaged in K' (right hand panel), and a gray scale map of the $J-K$ color in the same region (left hand panel). North is at the top, and east is to the left. The line in the lower left hand corner of the $K^{\prime}$ image subtends a spatial distance of 50 parsecs based on the Sakai \& Madore (2001) distance modulus. Redder colors appear lighter in the $J-K$ image. The CO sources R1 and R2 (Walter et al. 2002) coincide with areas of relatively red color; CO source R3 is located just off of the left hand edge of the displayed field. The smooth appearance of the $K^{\prime}$ image indicates that absorption from cool dust does not greatly affect the isophotal properties of NGC 3077 near $2 \mu \mathrm{m}$.

Fig. 3.- The $(J-H, H-K)$ TCD of pixels in the central $12 \times 14$ arcsec of NGC 3077 (left hand panel) and the corresponding region of NGC 205 (right hand panel). The error bars in the lower right hand corner of each panel show the uncertainties in the photometric calibration. The NGC 205 measurements were obtained from images that were processed to simulate the appearance of this galaxy if viewed at the same distance and with the same angular resolution as NGC 3077. The solar neightborhood giant and dwarf sequences from Tables II and III of Bessell \& Brett (1988) are shown, along with the mean locations of dEs, BCDGs, and starburst/HII galaxies in the samples observed by James (1994), Thuan (1983), and Hunt et al. (2002); the error bars show the standard deviation of the galaxy colors in each sample. The open squares show $\mathrm{z}=0.020 \alpha=2.35, \mathrm{M}_{u p}=100 \mathrm{M}_{\odot}$ instantaneous burst models from Leitherer et al. (1999), labelled according to $\log \left(\mathrm{t}_{y r}\right)$. The locus of the NGC 3077 data parallels the reddening vector, and the dispersion in the NGC 3077 data is suggestive of differential absorption of amplitude $\Delta \mathrm{A}_{V}=4$ magnitudes. An extrapolation along the reddening vector indicates that the light from the central regions of NGC 3077 has an unreddened SED that is similar to that of a simple stellar system with an age $\log \left(\mathrm{t}_{y r}\right)<6.8$. For comparison, the majority of pixels near the center of NGC 205 have an SED similar to that of a 'typical' Virgo cluster dE.

Fig. 4. - The $(J-H, H-K)$ TCD of pixels in the central $12 \times 14 \operatorname{arcsec}$ of NGC 3077 . 
The error bars in the lower right hand corner of each panel show the uncertainties in the photometric calibration. The solid lines show the result of combining light from NGC 205 with the instaneous burst $\mathrm{z}=0.020, \alpha=2.35$, and $\mathrm{M}_{u p}=100 \mathrm{M}_{\odot}$ Leitherer et al. (1999) models with $\log \left(\mathrm{t}_{y r}\right)=6.0$ (left hand panel) and 6.8 (right hand panel). Models with $\mathrm{A}_{V}=0$ magnitudes and $\mathrm{A}_{V}=6.0$ magnitudes from a uniform foreground absorbing sheet are shown to demonstrate the effects of reddening. The ratio of light from the young population to an NGC 205-like population in $K$ is indicated at various points along the model sequences. These models indicate that a significant fraction of the near-infrared light near the center of NGC 3077 must come from a relatively young population.

Fig. 5.- The surface brightness, ellipticity, and position angle profiles of NGC 3077 (left hand column) and NGC 205 (right hand column), as measured in $K^{\prime}$. The NGC 205 measurements are from images of this galaxy that have been processed to simulate its appearance if viewed at the same distance and with the same angular resolution as NGC 3077. The dashed line marks the approximate extent of the seeing disk, and the measurements to the left of this line are blurred by seeing. In both galaxies the central $K$ surface brightness is roughly 1 mag $\operatorname{arcsec}^{-2}$ brighter than would be expected if the surface brightness profile at larger radii were extrapolated to smaller radii. The error bar in the lower right hand corner of the panel containing the NGC 3077 surface brightness measurements shows the estimated \pm 0.1 magnitude $(1-\sigma)$ error in the last point due to uncertainties in the sky level.

Fig. 6. - The $(K, H-K)$ and $(K, J-K)$ CMDs of foreground stars and possible star clusters in NGC 3077, identified using the photomeric criteria discussed in $\S 4$. Objects identified as classical globular clusters and young clusters are plotted as filled and open squares, respectively. Likely foreground stars are plotted as stars. Objects within 15 arcsec of the galaxy center, which are marked with a cross, have the reddest colors at a given $K$, as expected if the amount of dust absorption increases with decreasing distance from the center of NGC 3077. The absolute magnitude calibration on the right hand axis assumes a distance modulus $\mu_{0}=27.9$ (Sakai \& Madore 1999).

Fig. 7.- The $(H-K, J-H)$ TCD of possible star clusters in NGC 3077. The symbols are the same as in Figure 6. The error bar shows the estimated uncertainty in the photometric calibration. The solid lines show the sequences for solar neighborhood giants and dwarfs from Tables II and III of Bessell \& Brett (1988). The dashed lines mark the area containing the bulk of classical globular clusters in M31, which are plotted as open triangles using data from Tables 1 of Barmby et al. (2000, 2001), extended to account for possible line of sight extinction in NGC 3077. The objects in the NGC 3077 data that fall within this area, and were not identified as foreground stars based on their colors and brightnesses (§4.1), are identified as globular clusters. The objects falling to the right of the globular cluster region 
are identified as possible young clusters. The filled triangles are $\mathrm{z}=0.020$ Leitherer et al. (1999) instanteous burst models with $\alpha=2.35$ and $\mathrm{M}_{u p}=100 \mathrm{M}_{\odot}$, which are labeled with $\log \left(\mathrm{t}_{y r}\right)$. The majority of the candidate young clusters have SEDs consistent with reddened versions of the $\log \left(\mathrm{t}_{y r}\right)=6.0$ and 6.6 models. The approximate midpoints of the regions containing the candidate globular clusters and young clusters are labelled with 'GC' and 'YC', respectively. 


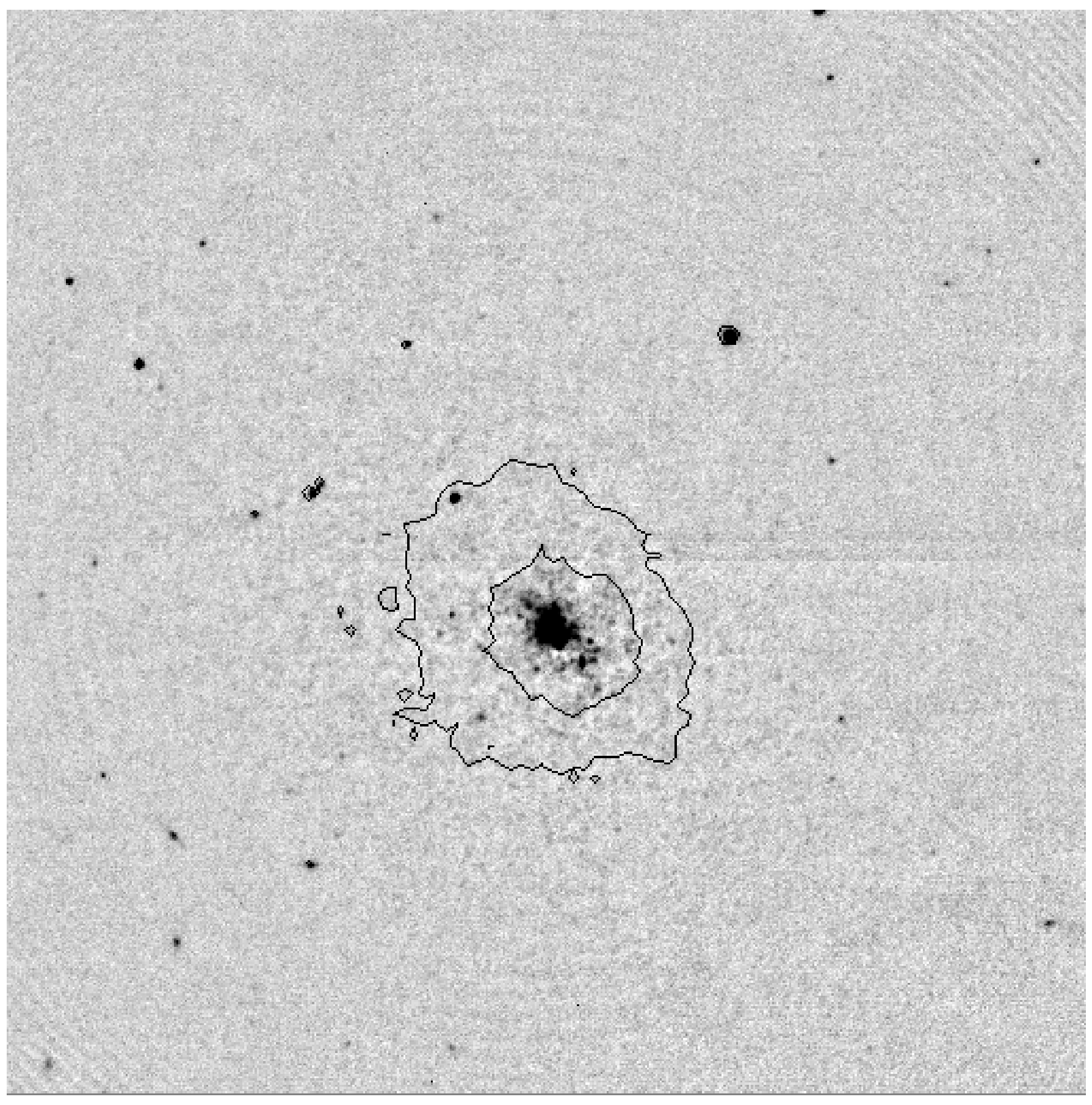



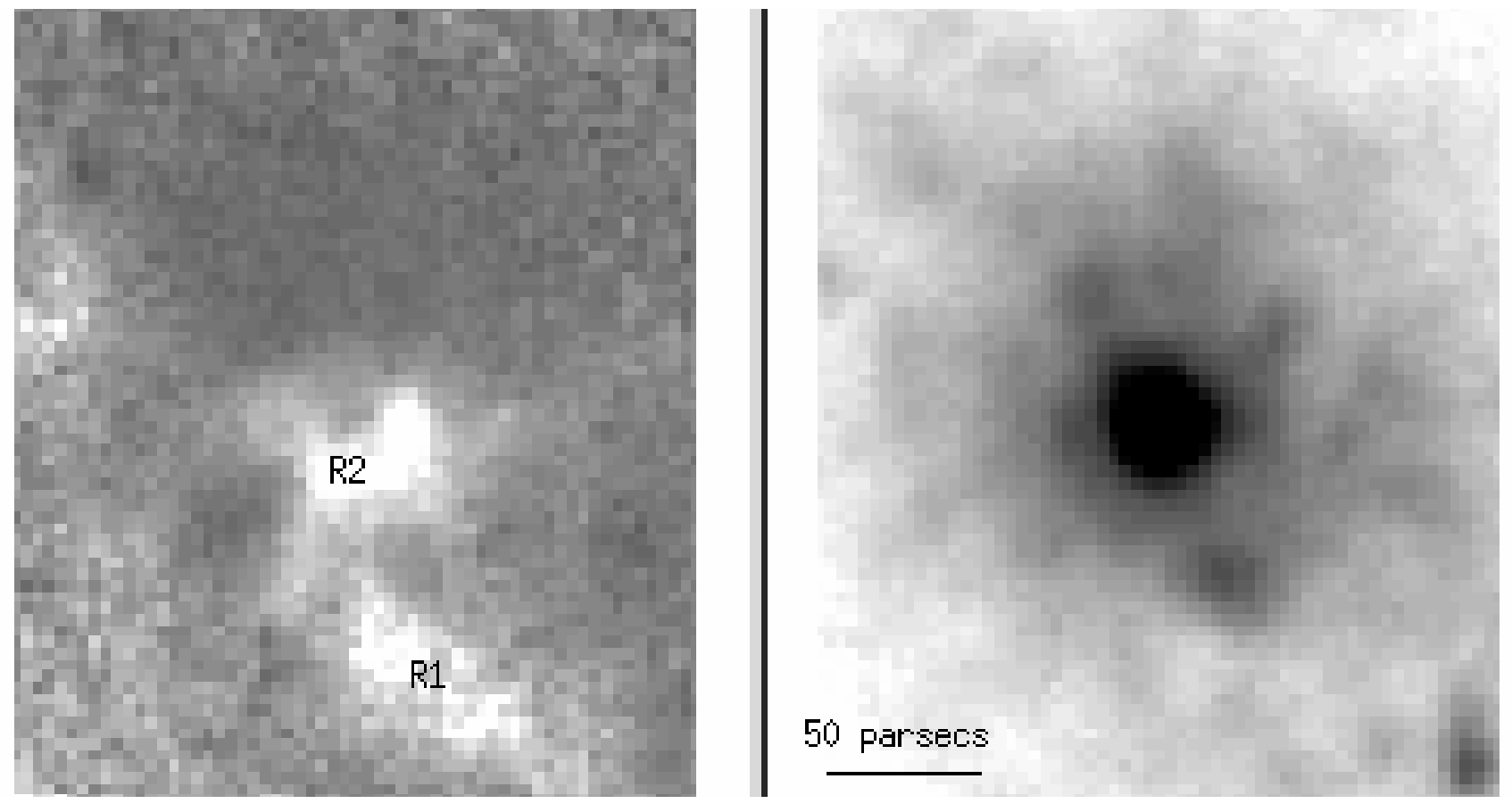


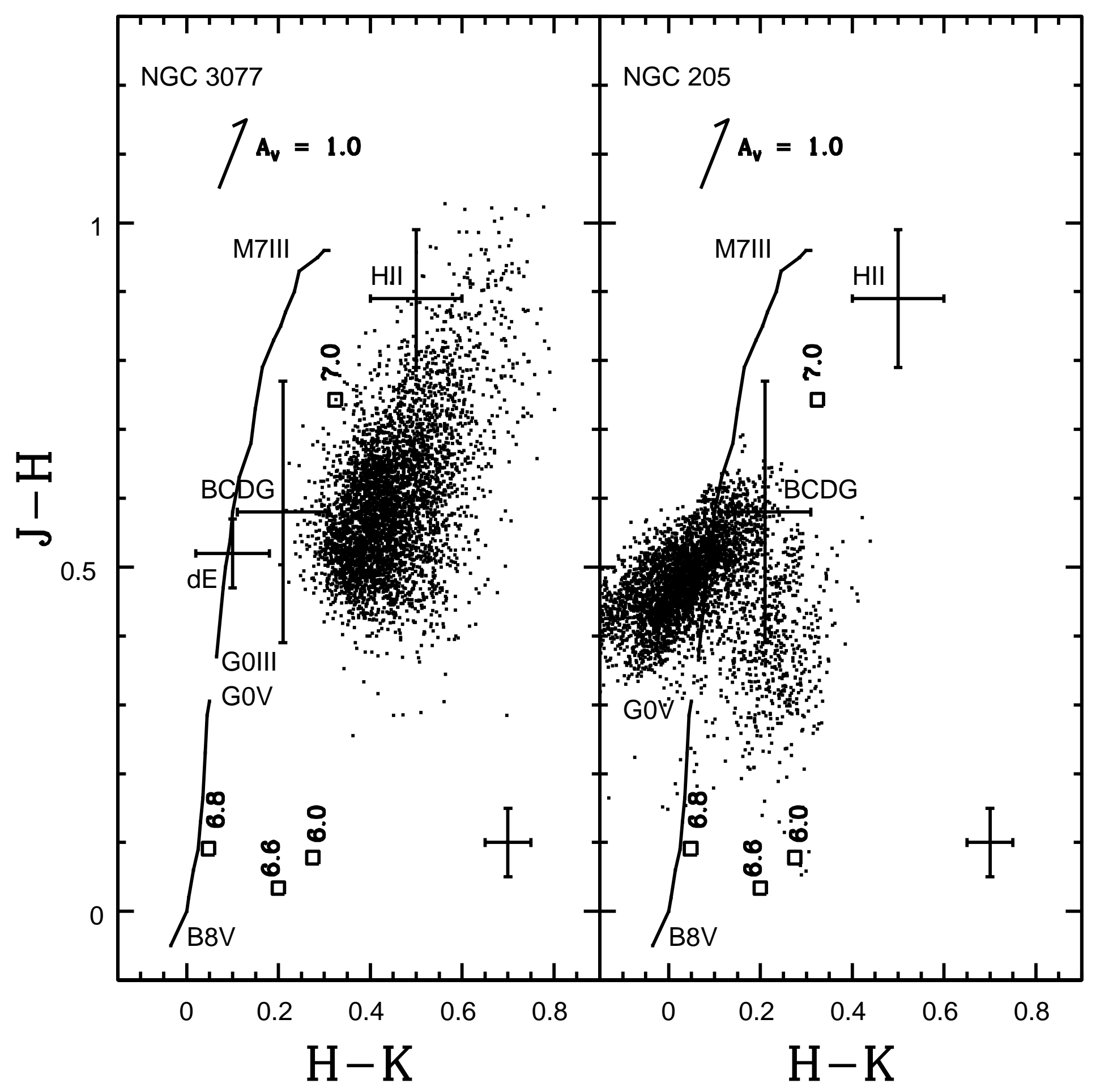




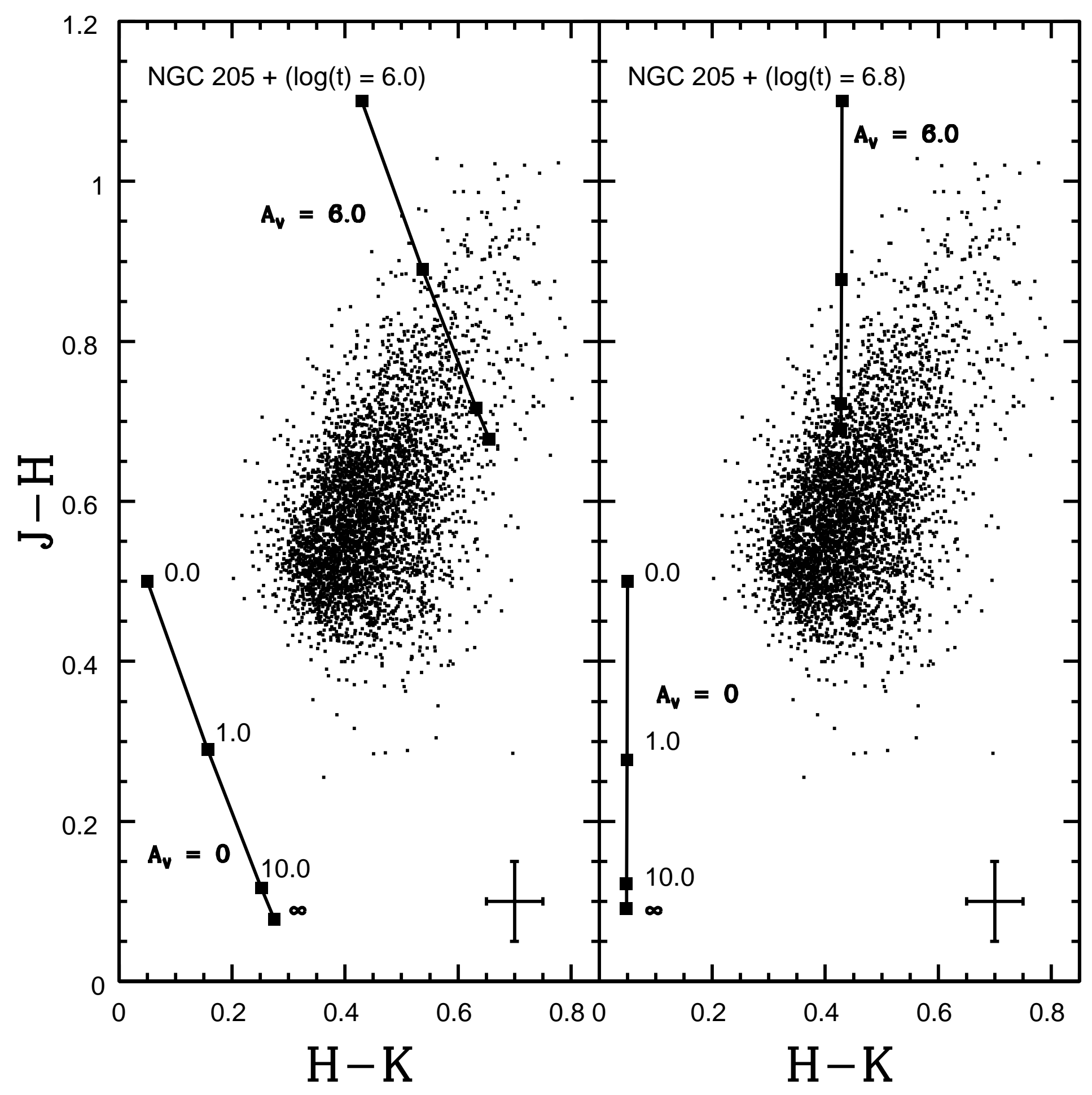




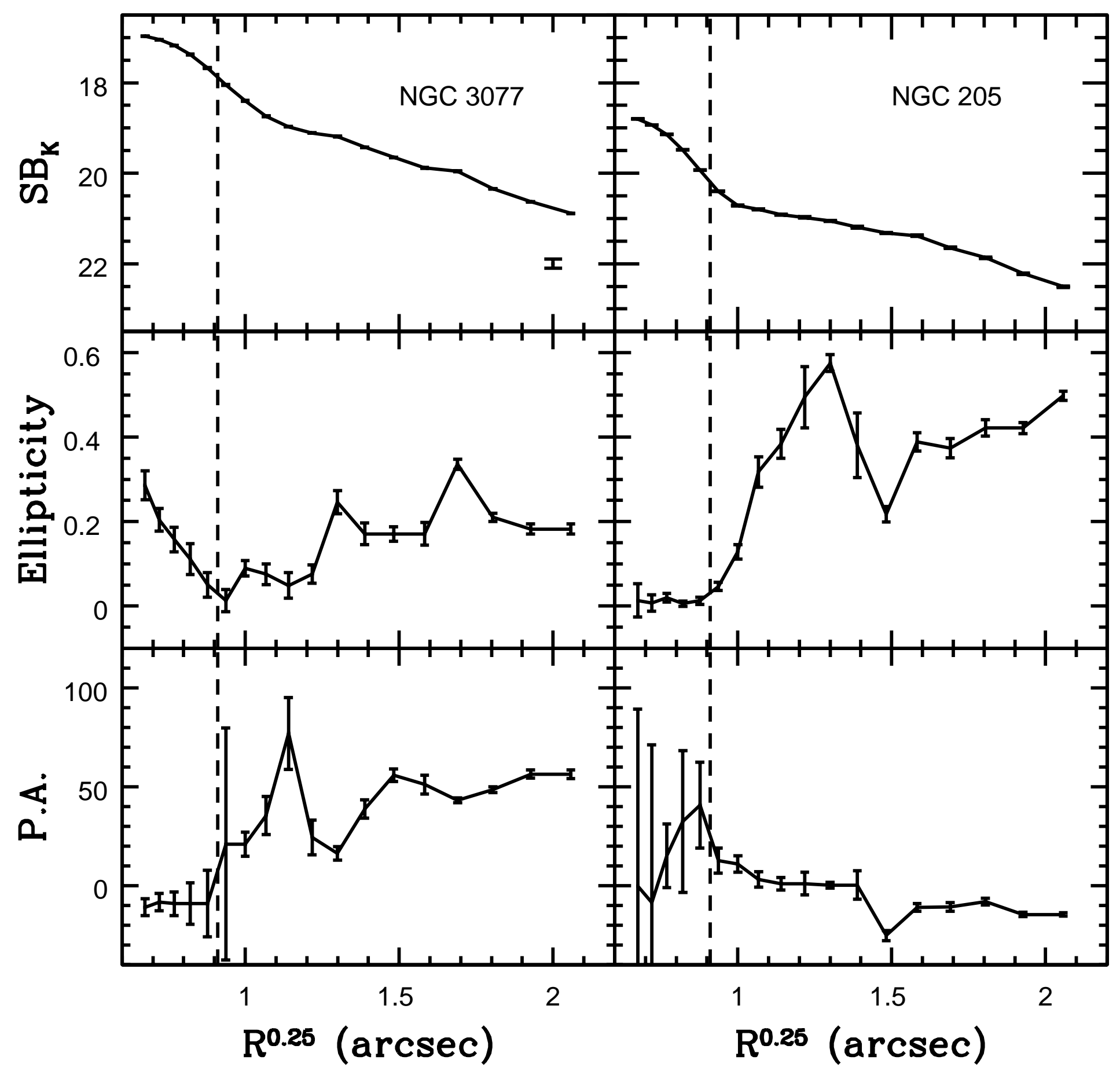




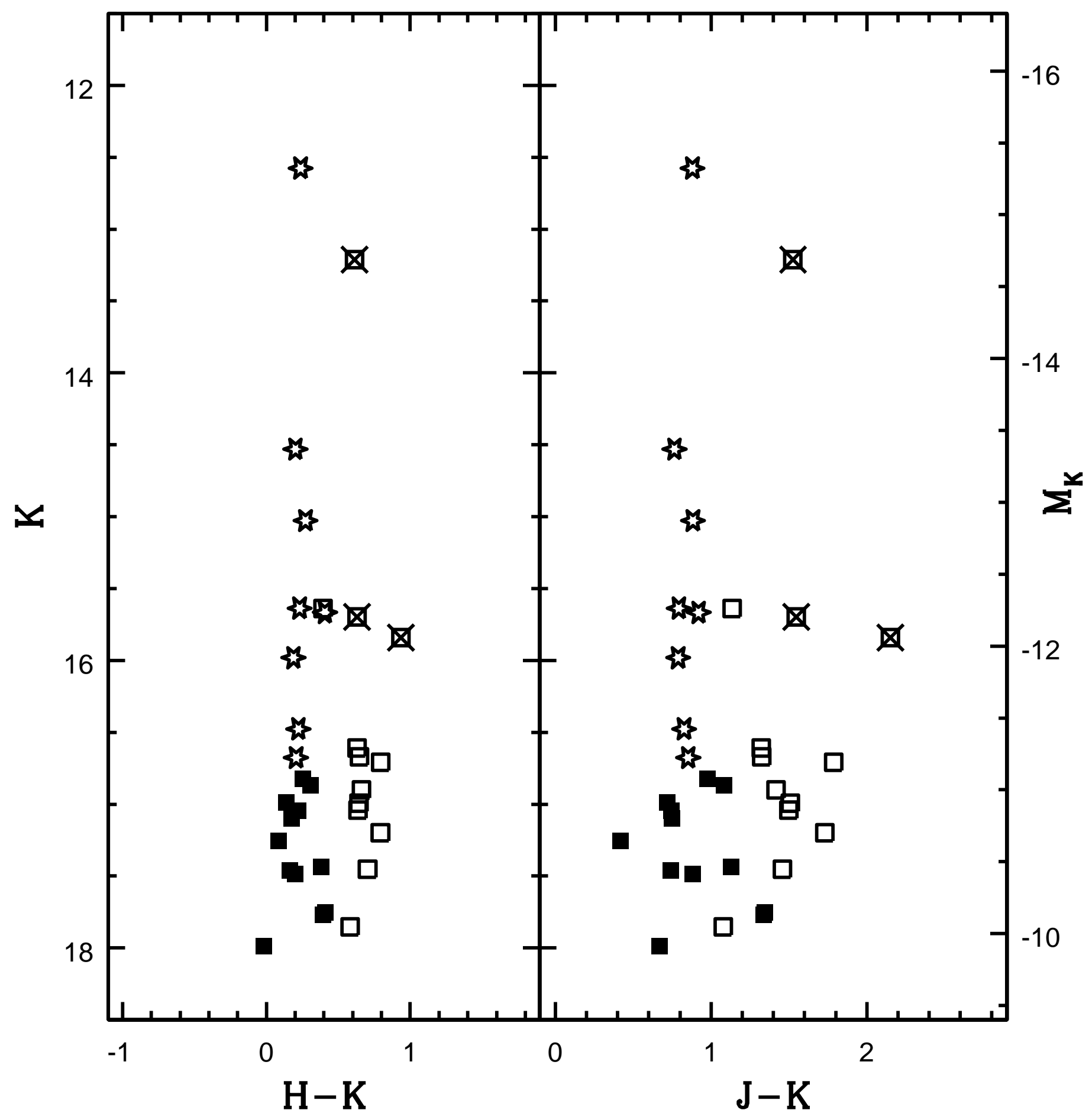




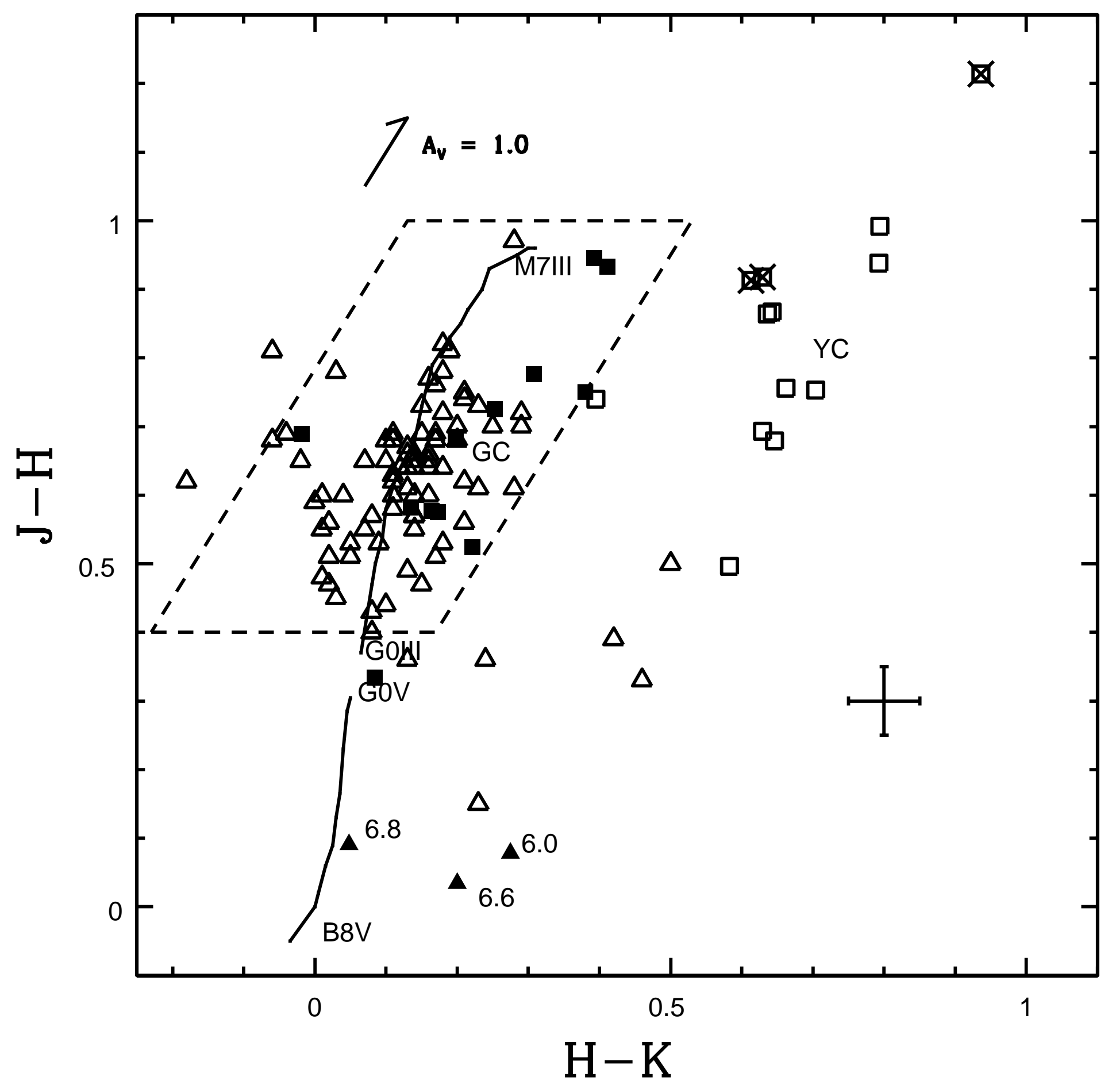

